\title{
Experimental investigation on pump efficiency and cavitation performance of Huang-jin-xia hydro-junction
}

\author{
Shaojiang Luo ${ }^{1}$, Tao Dong ${ }^{1}$, Yong Mou ${ }^{1}$, and Chenhao $\mathrm{Li}^{2 *}$ \\ ${ }^{1}$ HanJiang-To-WeiHe River Valley Water Diversion Project Construction Co.Ltd., Shaanxi Province , Xi'an, 710100, China \\ ${ }^{2}$ State Key Laboratory of Eco-hydraulics in Northwest Arid Reign of China, Xi'an University of Technology, Xi'an, 710048, China
}

\begin{abstract}
In this paper, the performance of A1515 centrifugal pump was studied on a high parameters and precision large general test platform in combination with the Huang-jin-xia hydro-junction in Hanjiang-toWeihe River valley water diversion project. The efficiency characteristics and cavitation performance of the pump were tested and measured respectively. All measured parameters were collected and recorded through the acquisition system. The experimental results show that the optimal efficiency and weighted average efficiency of the pump is $92.46 \%$ and $92.18 \%$, and the design point efficiency is $92.39 \%$, all of which meet the design requirements. After conversion to the prototype pump, the efficiency value at each working point is higher than the guaranteed value. The pump hump margin is $3.03 \%$, which is greater than $2 \%$ of the required value and meets the requirements. In the whole normal operating range, the cavitation performance of the model pump meets the design and test requirements. The comprehensive error of model test is less than $\pm 0.2 \%$. The research results provide reference for the design of large centrifugal pump.
\end{abstract}

\section{Introduction}

Centrifugal pump is widely used in industrial production because of its advantages of high head. Because the large pump technology is not mature interiorly, there is no relevant data reference, it is necessary to research the pump by model experiment ${ }^{[1]}$. The energy and efficiency performance of centrifugal pump unit is not only related to the degree of energy consumption, but also affects the safety of pump unit. It is of great significance to carry out the pump performance under different working conditions $^{[2-4]}$. Secondly, cavitation is a physical phenomenon in the operation of pump, and the cavitation process will produce large pressure pulsation. And cavitation collapse will produce a lot of impact and noise, resulting in the unit performance decline; therefore, it is very necessary to study the cavitation performance through experiments.

At present, researchers have done a lot of research on pumps' cavitation. Lin $^{[5]}$ conducted numerical calculation on LES turbulence model and cavitation model for the whole flow passage, and concluded that the draft tube was affected by the runner inlet cavitation, and the amplitude of pulsation frequency increased. Zhao et al. ${ }^{[6]}$ studied the pressure pulsation law and the change law of cavitation volume in a low specific speed centrifugal pump during cavitation, the results showed that the bubble volume increased non-linearly in the cavitation process. Luo et al. ${ }^{[7]}$ studied the characteristics of the centrifugal pump's transient speed under different conditions by experiments. When cavitation just happened, the pump's speed varied in the largest range, and the degree of rotation speed fluctuation under severe cavitation is higher than that without cavitation. Wang et al. ${ }^{[8]}$ simulated the transient cavitation flow in a centrifugal pump based on the RNG $\mathrm{k}-\varepsilon$ turbulence model and the improved transport cavitation model. Zhao ${ }^{[9]}$ studied the positive and negative problems of cavitation control by placing obstacles on the blade surface of a centrifugal pump through experiments and numerical simulation. The results show that the head value of pump can be increased by $30 \%$ when the blade pressure side is placed with obstacles.

This paper takes the centrifugal pump of Huangjinxia hydro-junction as the research object and carries out the model pump experiment. The cavitation performance of pump is studied by visualization, and the energy and efficiency characteristics of pump under different speed and flow conditions was carried out, finally, the characteristic curve of the pump was drawn, the cavitation performance of pump with different cavitation numbers was analysed.

\section{Pump model and experimental apparatus}

Model pumps including inlet pipe, volute, stay ring, head cover, impeller et al. The model device is made of metal materials except for the straight conical section of the inlet pipe which is transparent Perspex, the rest are metal materials. The whole flow channel of the pump from inlet pipe to volute outlet is geometrically similar to the prototype pump, the scale coefficient of the prototype and

${ }^{*}$ Corresponding author: drleech_xaut@126.com 
the model is $\mathrm{D} 1 \mathrm{p} / \mathrm{D} 1 \mathrm{~m}=4.551$. Fig. 1 shows a schematic diagram of the pump model device. Table 1 shows the basic parameters of the test model pump. All test parameters meet the requirements of relevant standards of GB15613-2008 and IEC60193-1999. The measurement accuracy and operation stability of all test parameters are required. The comprehensive test error of the model efficiency of the test bench is less than $\pm 0.20 \%$.

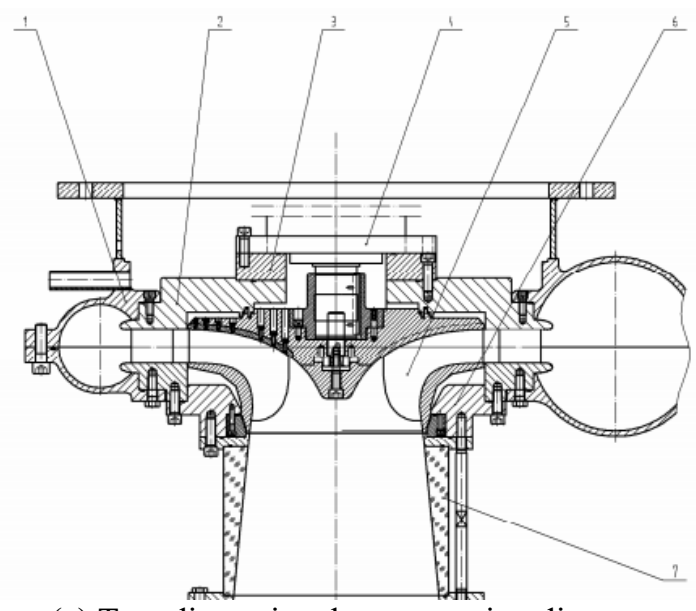

(a) Two dimensional cross-section diagram

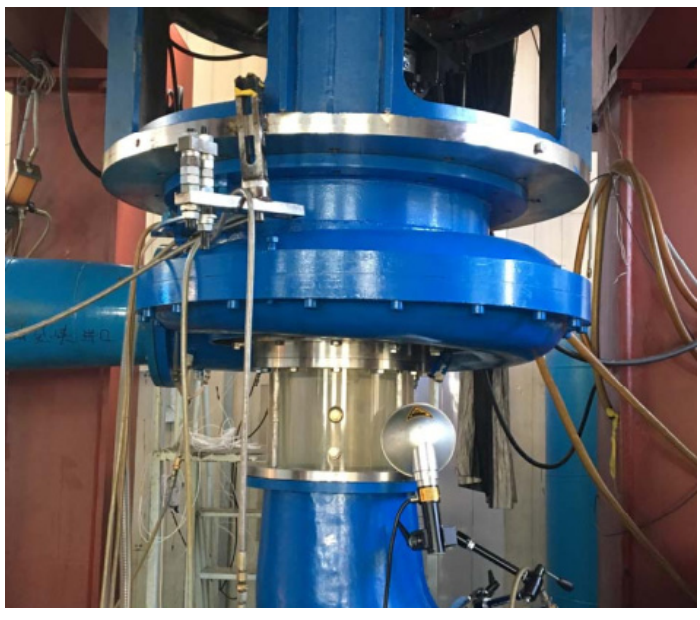

(b) Experimental device diagram

Figure 1. Caption of the Figure 1. Below the figure. 1-volute; 2-head cover; 3-sub-plate; 4-binder-plate;

5-impeller; 6- stay ring; 7-inlet taper pipe;
Table1. Main parameters of model pump

\begin{tabular}{cc}
\hline Designations & Parameters \\
\hline Pump type & $\mathrm{A} 1515$ \\
Pump inlet diameter & $\mathrm{D}_{2 \mathrm{~m}}=0.307 \mathrm{~m}$ \\
Pump outlet & $\mathrm{D}_{1 \mathrm{~m}}=0.529 \mathrm{~m}$ \\
diameter & 9 \\
Blade number & $\mathrm{Z}_{\mathrm{s}}=13$ \\
Guide vane number & $\mathrm{Q}_{\mathrm{d}}=0.348 \mathrm{~m} 3 / \mathrm{s}$ \\
Design flow rate & $\mathrm{H}_{\mathrm{d}}=37.93 \mathrm{~m}$ \\
Design head & $\mathrm{n}_{\mathrm{m}}=1000 \mathrm{r} / \mathrm{min}$ \\
Rated speed &
\end{tabular}

\section{Results and analysis}

\subsection{Analysis of efficiency experimental results}

Efficiency test is to measure the efficiency, flow rate, head, power and other parameters of the pump under the water condition. Including the efficiency characteristics of the normal frequency variation range $49.8 \sim 50.5 \mathrm{~Hz}$ and the head range. In the test, set the dynamometer speed as $\mathrm{n}_{\mathrm{m}}=1000 \mathrm{r} / \mathrm{min}$. When drawing model pump characteristic curve, horizontal axis represents flow rate vertical axis represents head, pump efficiency and shaft power, andthe actual operating area of prototype pump should be marked on the pump characteristic curve. The formula for calculating the weighted average efficiency of pump is as follows:

$$
\eta_{\text {ave }}=\sum_{j=1}^{n} \omega_{j} \cdot \eta_{j} / 100
$$

Where, $\omega_{j}$ and $\eta_{j}$ are efficiency weighting factor and efficiency, respectively.

According to the test requirements, when the model pump speed is $\mathrm{nm}=1000 \mathrm{r} / \mathrm{min}$ and the power frequency is $50 \mathrm{~Hz}$, the efficiency tests are carried out on the optimal and weighted average points of the pump under 6 groups of different head. The efficiency test results are shown in Table 2. In addition, the flow-head curve test was carried out in two ways of flow increasing and flow decreasing under the same speed condition. Finally, in the range of $100 \% \mathrm{~N}_{\mathrm{r}} \sim 92 \% \mathrm{~N}_{\mathrm{r}}$, the pump efficiency test at different speeds is carried out according to $1 \% \mathrm{~N}_{\mathrm{r}}$ interval. The results are shown in Fig. 2 and Fig. 3.

Table2. Weighted average efficiency of model A1515

\begin{tabular}{|c|c|c|c|c|c|c|}
\hline \multirow{2}{*}{ Parameters } & \multicolumn{6}{|c|}{ Head (m) } \\
\hline & 101.7 & 104.5 & 106.5 & 108.5 & 110 & 116.5 \\
\hline Weighting coefficient & 10 & 31 & 38 & 14 & 3 & 4 \\
\hline Model head (m) & 34.9 & 35.86 & 36.52 & 37.26 & 37.73 & 39.92 \\
\hline Mode flow rate $\left(\mathrm{m}^{3} / \mathrm{s}\right)$ & 0.3868 & 0.3745 & 0.3658 & 0.357 & 0.3504 & 0.3214 \\
\hline Model efficiency (\%) & 91.74 & 92.08 & 92.25 & 92.39 & 92.44 & 92.37 \\
\hline Prototype efficiency (\%) & 92.74 & 93.08 & 93.25 & 93.39 & 93.44 & 93.37 \\
\hline Prototype head (m) & 101.73 & 104.57 & 106.53 & 108.58 & 110.01 & 116.51 \\
\hline Prototype flow rate $\left(\mathrm{m}^{3} / \mathrm{s}\right)$ & 13.66 & 13.23 & 12.93 & 12.61 & 12.38 & 11.36 \\
\hline \multirow[t]{2}{*}{ Input power (MW) } & 14.66 & 14.54 & 14.45 & 14.34 & 14.26 & 13.87 \\
\hline & \multicolumn{3}{|c|}{ Experimental value } & \multicolumn{3}{|c|}{ Guarantee value } \\
\hline Model Weighted average efficiency (\%) & \multicolumn{3}{|c|}{92.18} & \multicolumn{3}{|c|}{-} \\
\hline Prototype Weighted average efficiency (\%) & \multicolumn{3}{|c|}{93.18} & \multicolumn{3}{|c|}{$\geq 93.14$} \\
\hline
\end{tabular}




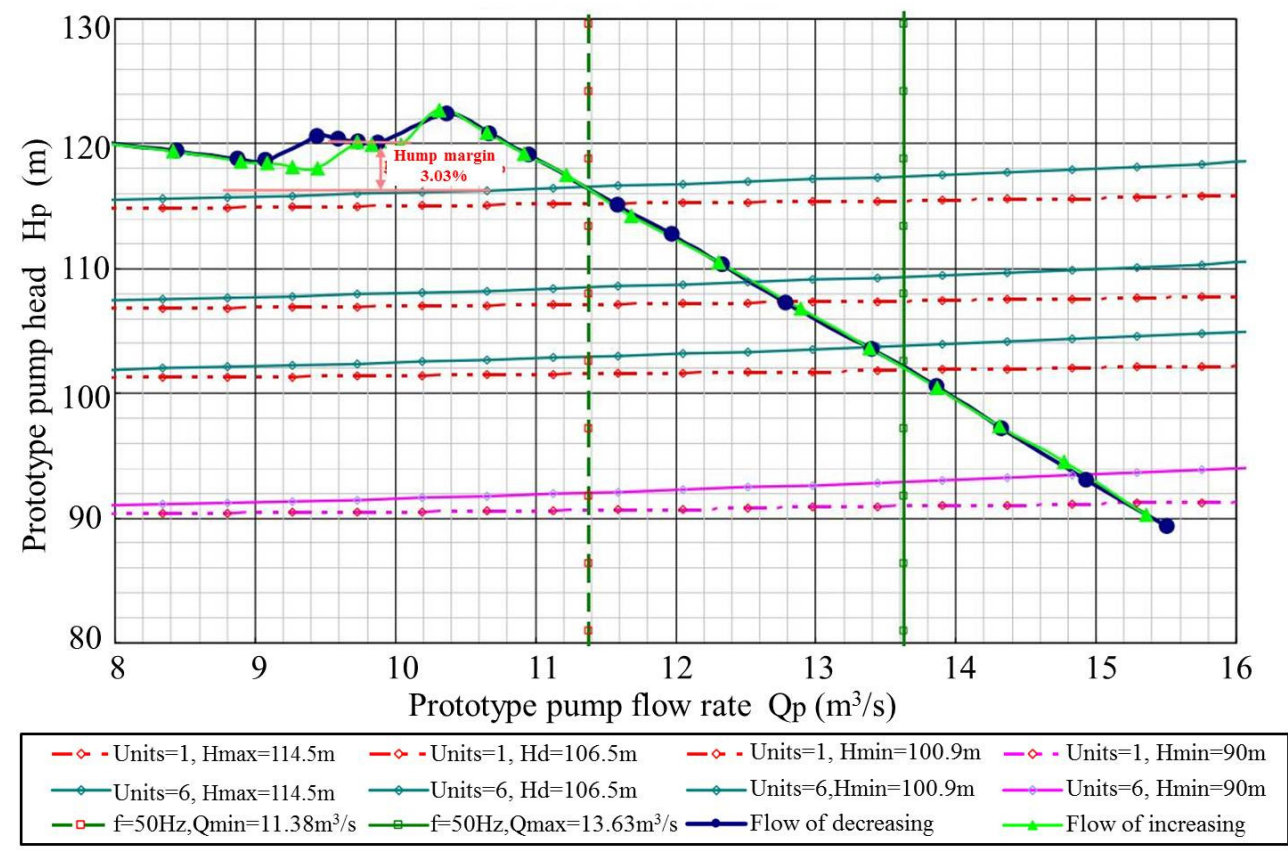

Figure 2. A1515 prototype pump energy characteristic curve $\left(\mathrm{n}_{\mathrm{rp}}=375 \mathrm{r} / \mathrm{min} \mathrm{f}_{0}=50 \mathrm{~Hz} \mathrm{D}_{\mathrm{p}}=1.397 \mathrm{~m}\right)$

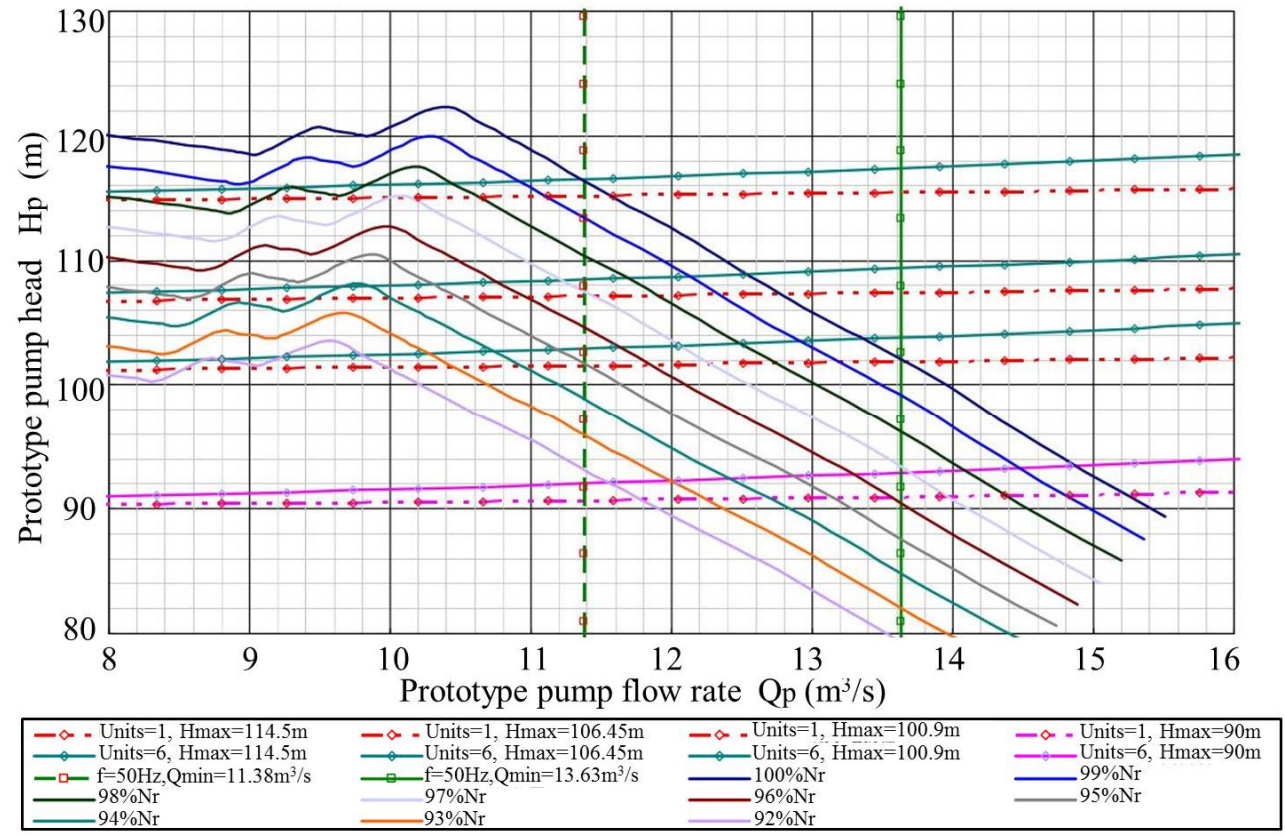

Figure 3. Energy characteristic curve of prototype pump at different rotating speeds

\subsection{Cavitation performance experimental results analysis}

According to the test requirements, the cavitation performance of the pump was carried out, and the results were recorded and analysed. The pump condition cavitation test is carried out under the specified head, input power and frequency (including abnormal frequency). The Cavitation tests were conducted under different heads with cavitation numbers, including critical cavitation tests and determination of primary cavitation coefficients.
The cavitation test of the model pump needs to determine the primary cavitation $N P S H_{i}$ and critical cavitation $N P S H_{1.0 \%}$ at different conditions, and indicate the $N P S H_{P}$ of the pump station device, which met the normal operation conditions of the pump station, the pump station cavitation $N P S H_{P}$ is greater than the primary cavitation NPSHi. The critical cavitation $\mathrm{NPSH}_{1.0 \%}$ value is defined as the cavitation NPSH value when the suction height decreases and the efficiency is lower than the efficiency of $1.0 \%$ in the no-cavitation condition. Primary cavitation $N P S H_{i}$ value is defined as the cavitation NPSH value when stable bubbles were 
attached on two blades of the impeller by visual observation. Pumping unit cavitation coefficient is $\sigma_{p}=$ $N P S H_{P} / H_{P}$; primary cavitation coefficient is $\sigma_{i}=$ $\mathrm{NPSH}_{i} / \mathrm{H}_{P}$; critical cavitation coefficient is $\sigma_{1.0 \%}=$ $\mathrm{NPSH}_{1.0 \%} / \mathrm{H}_{P}$.

The test results in Fig. 4 and Fig. 5 show that within the normal operation range of the pump, the pump device's net positive suction head station $N P S H_{P}$ is greater than the primary cavitation $N P S H_{i}$, and the pump station's cavitation safety factors $K_{N P S H_{i}} \geq 1.2$, $K_{N P S H_{1.0 \%}} \geq 1.6$, both meet the requirements.

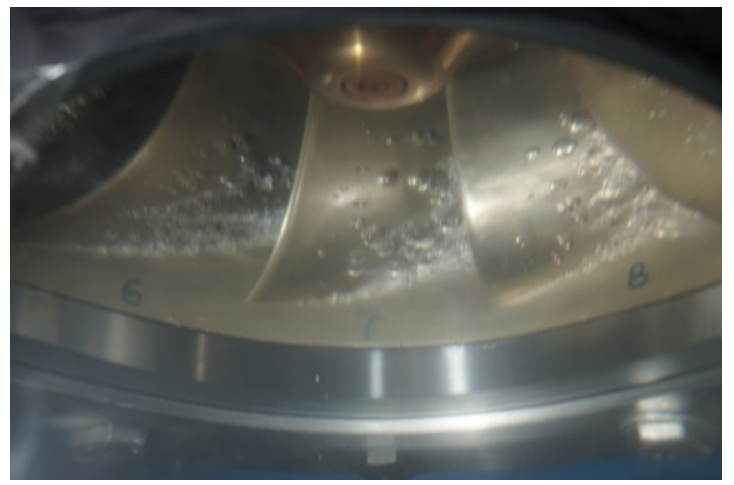

(a) $\mathrm{H}_{\mathrm{p}}=101.6 \mathrm{~m}, 100 \% N_{\mathrm{r}}$

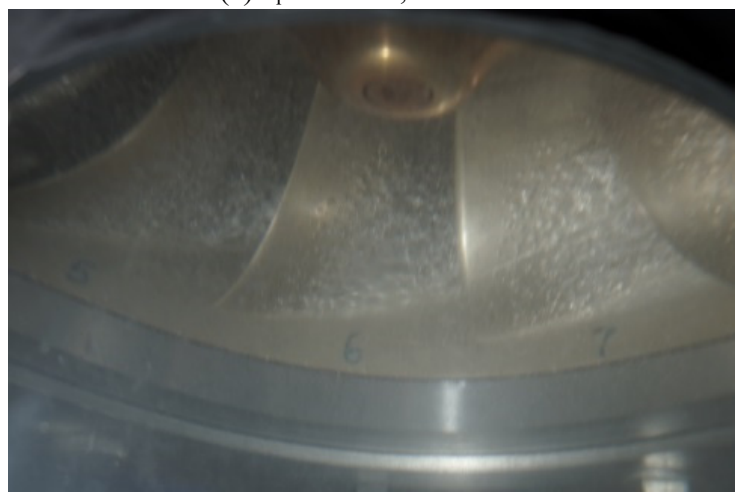

(c) $\mathrm{Hp}=108.5 \mathrm{~m}, 100 \% \mathrm{Nr}$

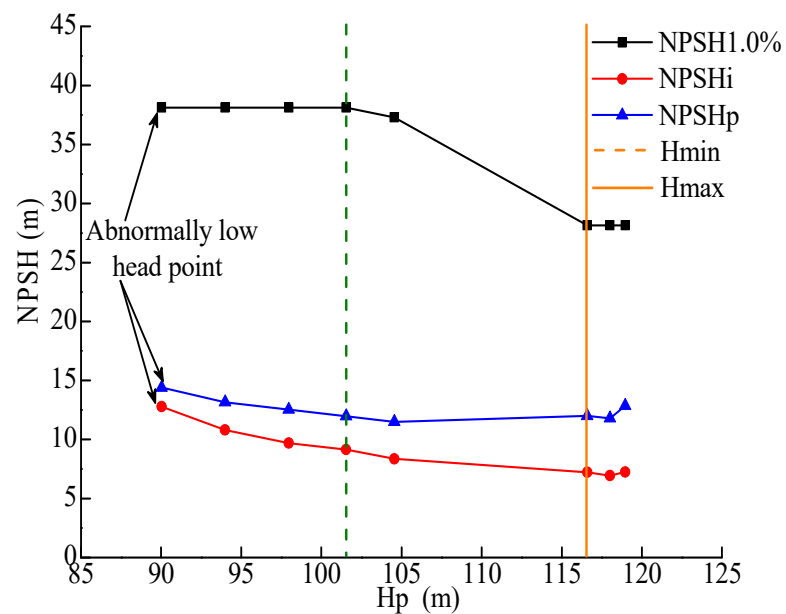

Figure 4. Cavitation experimental results of A1515 prototype pump

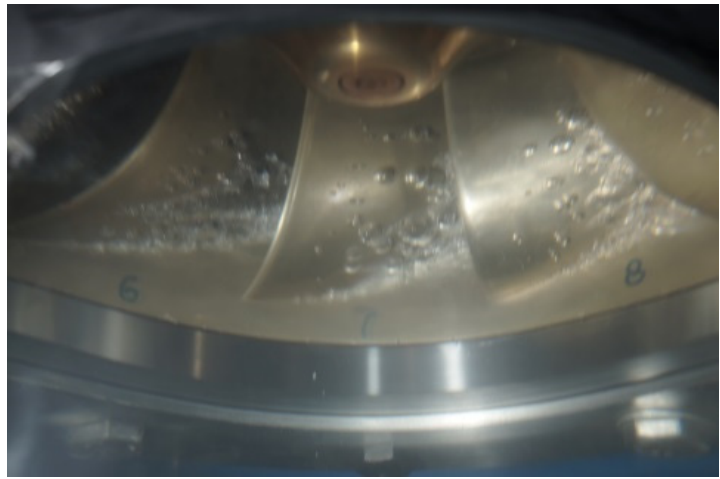

(b) $\mathrm{H}_{\mathrm{p}}=106.5 \mathrm{~m}, 100 \% N_{\mathrm{r}}$

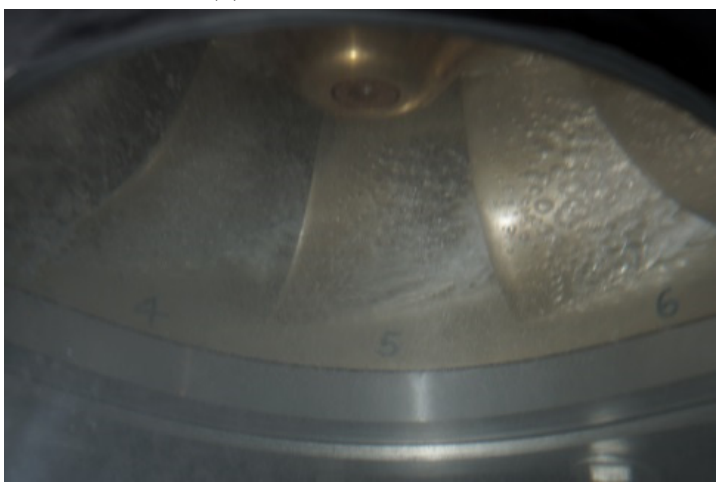

(d) $\mathrm{Hp}=116.5 \mathrm{~m}, 100 \% \mathrm{Nr}$

Figure 5. Cavitation shape of the prototype pump at critical cavitation $(\sigma 1.0 \%)$ under different heads

\section{Conclusions}

Based on the high-precision and high-parameters pump test platform, this paper conducts experimental research on the efficiency characteristics and cavitation performance of A1515 model pump under different working conditions through fine test methods, and analyses the efficiency and cavitation characteristics of pump under different working conditions. The main conclusions are as follows:

(1) The optimal efficiency, weighted average efficiency and design efficiency of the model pump are $92.46 \%, 92.18 \%$ and $92.39 \%$ respectively, all of which meet the design requirements. After the conversion to the prototype pump, the efficiency values at each condition are higher than the guaranteed value, which indicates that the design indexes of the pump meet the contract requirements.

(2) A1515 model pump can meet the contract requirements at the minimum flow rate and design head flow rate, and the design flow rate is close to the optimal condition. Pump hump margin is $3.03 \%$, which is greater than the required $2 \%$ and meet the requirements.

(3) Within the whole normal operating range, the cavitation performance of the A1515 model pump with different cavitation numbers meets the design and test requirements. 


\section{Acknowledgements}

This study was supported by the Science and Technology Innovation Fund Project of Hanjiang-to-Weihe River Valley Water Diversion Project Construction Co. Ltd. "Study on the outage and escape transition process of centrifugal pump under the influence of long water pipeline" (NO: 2020203) and Open project of State Key Laboratory of Hydroelectric Equipment (NO: SKLHEORF-202004)

\section{References}

1. Y Zhang, D Zhang, Y Liu, et al. Fluid Machinery, 31 1 (2003),.

2. B Chen,B Zhen, $\mathrm{H}$ Zhang, et al. China Water \& Wastewater, 35,91-95(2019).

3. H Xie,Z LI, D Liu, S Huang. China Rural Water and Hydropower, 91,29 (2019).

4. R Tao, R Xiao, W Yang. Journal of Drainage and Irrigation Machinery Engineering, 36,573(2018).

5. S Lin. Study on the influence of cavitation on the hump characteristics of pump turbine, Harbin Institute of Technology,(2019).

6. W Zhao, G Wang, G Zhao, et al. Journal of Lanzhou University of Technology, 46,50 (2020).

7. Y Luo, J Dong, Y Han, et al. Journal of vibration and shock, 40,23 (2021).

8. W Wang, Q Meng, $\mathrm{H} \mathrm{Lu}$, et al. China Rural Water and Hydropower, 92,202 (2017).

9. G Zhao. Investigation of Cavitation instabilities and it's Control in a Centrifugal pump, Lanzhou University of Technology, (2018). 